\title{
Distributed Joint Receiver Design for Uplink Cell-Free Massive MIMO
}

\author{
Italo Atzeni, Bikshapathi Gouda, and Antti Tölli \\ Centre for Wireless Communications, University of Oulu, Finland \\ Emails: \{italo.atzeni, bikshapathi.gouda, antti.tolli\}@oulu.fi
}

\begin{abstract}
The majority of works on cell-free massive multipleinput multiple-output (MIMO) adopt uncoordinated transmit/receive strategies at the base stations (BSs) to avoid extensive channel state information (CSI) exchange via backhaul signaling. However, significant performance gains can be achieved by allowing coordination among the BSs. This paper proposes a distributed joint receiver design for uplink cell-free massive MIMO based on over-the air (OTA) signaling, which entirely eliminates the need for backhaul signaling for CSI exchange. The proposed scheme is flexible and scalable, as the amount of OTA signaling does not grow with the number of BSs or user equipments. Numerical results show fast convergence and remarkable performance gains with respect to uncoordinated joint reception.
\end{abstract}

\section{INTRODUCTION}

Cell-free massive multiple-input multiple-output (MIMO) is a recently coined concept that suitably combines elements from massive MIMO, small cells, and user-centric coordinated multi-point (CoMP) with joint transmission/reception [1][3]. In cellular massive MIMO networks, each base station (BS) is equipped with a very large antenna array and serves numerous user equipments (UEs) simultaneously by means of highly directional beamforming techniques [4]. In the cell-free context, the massive MIMO regime is achieved by spreading a large number of antenna elements across the network, which provides overall enhanced coverage and reduced pathloss. In addition, cell-free massive MIMO systems are characterized by a user-centric joint transmission/reception extended to the whole network such that there are no cell boundaries and each UE is served jointly by all the BSs [5].

Cell-free massive MIMO has been the subject of a broad literature over the past few years and is now regarded as a potential physical-layer paradigm shift for beyond-5G systems. Remarkably, cell-free massive MIMO networks have been shown to outperform their traditional cellular massive MIMO and small-cell counterparts in several scenarios of practical interest [1], [6], [7]. To avoid cumbersome and costly channel state information (CSI) exchange among the BSs via backhaul signaling and to reduce the overall computational complexity, most works on cell-free massive MIMO assume simple uncoordinated transmit/receive strategies at the BSs, such as maximum ratio transmission/combining, local zero-forcing, and local minimum mean squared error (MMSE), which can be implemented based on local CSI. Nevertheless, the performance of cell-free massive MIMO systems can be sensibly boosted by increasing the level of coordination among the BSs [7].

This work was supported by the Academy of Finland under grant no. 318927 (6Genesis Flagship).
Despite the potential gains of cooperative processing in CoMP, both centralized and distributed precoding/combining approaches have not found their way into practical implementation due the enormous amount of required CSI exchange via backhaul signaling, either between the BSs and a central processing unit (CPU) or among the BSs [8]. Indeed, this represents an even greater obstacle in a cell-free massive MIMO context due to the large number of BSs and UEs involved in the network-wide processing. Furthermore, the backhaul introduces delays and quantization errors in the CSI exchange that can lead to further performance losses. In our recent work [9], we proposed a distributed framework for cooperative precoding design in downlink cell-free massive MIMO systems that entirely eliminates the need for backhaul signaling for CSI exchange. This is based on an innovative over-the-air (OTA) signaling mechanism whereby each BS can obtain the missing cross-terms necessary for the distributed precoding design via bi-directional signaling between the BSs and the UEs.

In this paper, we focus on the uplink scenario and propose a novel distributed joint receiver design for cell-free massive MIMO. Exploiting time division duplex (TDD) operations, an OTA signaling mechanism similar to the one introduced in [9] is adopted to avoid CSI exchange among the BSs via backhaul signaling. The proposed distributed joint receiver design enjoys desirable flexibility and scalability properties, as the amount of OTA signaling does not scale with the number of BSs or UEs. Numerical results show very fast convergence and remarkable performance gains in terms of average sum rate with respect to the (uncoordinated) local MMSE receiver.

\section{SySTEM MODEL}

We consider a cell-free massive MIMO system where a set of $M$-antenna BSs $\mathcal{B} \triangleq\{1, \ldots, B\}$ serves a set of $N$-antenna UEs $\mathcal{K} \triangleq\{1, \ldots, K\}$ in the uplink. Assuming a TDD setting and a single data stream transmitted by each UE, let $\mathbf{H}_{b, k} \in$ $\mathbb{C}^{M \times N}$ denote the uplink channel matrix between UE $k \in \mathcal{K}$ and BS $b \in \mathcal{B}$, with $\mathbf{H}_{k} \triangleq\left[\mathbf{H}_{1, k}^{\mathrm{T}}, \ldots, \mathbf{H}_{B, k}^{\mathrm{T}}\right]^{\mathrm{T}} \in \mathbb{C}^{B M \times N}$ being the aggregated uplink channel matrix seen by UE $k$. Moreover, let $\mathbf{w}_{k} \in \mathbb{C}^{N \times 1}$ denote the precoding vector used by UE $k$, with $\left\{\left\|\mathbf{w}_{k}\right\|^{2} \leq \rho_{\mathrm{UE}}\right\}_{k \in \mathcal{K}}$. The receive signal at $\mathrm{BS} b$ is given by

$$
\mathbf{y}_{b} \triangleq \sum_{k \in \mathcal{K}} \mathbf{H}_{b, k} \mathbf{w}_{k} d_{k}+\mathbf{z}_{b} \in \mathbb{C}^{M \times 1}
$$

where $d_{k} \sim \mathcal{C N}(0,1)$ is the transmit data symbol of UE $k$ and $\mathbf{z}_{b} \in \mathbb{C}^{M \times 1}$ is the average white Gaussian noise (AWGN) term at $\mathrm{BS} b$, with elements distributed as $\mathcal{C N}\left(0, \sigma_{\mathrm{BS}}^{2}\right)$. Likewise, the 
aggregated receive signal at all the BSs is given by

$$
\mathbf{y} \triangleq \sum_{k \in \mathcal{K}} \mathbf{H}_{k} \mathbf{w}_{k} d_{k}+\mathbf{z} \in \mathbb{C}^{B M \times 1}
$$

with $\mathbf{z} \triangleq\left[\mathbf{z}_{1}^{\mathrm{T}}, \ldots, \mathbf{z}_{B}^{\mathrm{T}}\right]^{\mathrm{T}} \in \mathbb{C}^{B M \times 1}$. Let $\mathbf{v}_{b, k} \in \mathbb{C}^{M \times 1}$ denote the BS-specific combining vector used by BS $b$ for UE $k$, with $\mathbf{v}_{k} \triangleq\left[\mathbf{v}_{1, k}^{\mathrm{T}}, \ldots, \mathbf{v}_{B, k}^{\mathrm{T}}\right]^{\mathrm{T}} \in \mathbb{C}^{B M \times 1}$ being the aggregated combining vector used for UE $k$ (accordingly, we have $\sum_{b \in \mathcal{B}} \mathbf{v}_{b, k}^{\mathrm{H}} \mathbf{H}_{b, \bar{k}}=\mathbf{v}_{k}^{\mathrm{H}} \mathbf{H}_{\bar{k}}$ ). Each BS $b$ estimates $d_{k}$ by combining $\mathbf{y}_{b}$ with $\mathbf{v}_{b, k}$ and the estimates from all the BSs are collected by the CPU. The resulting signal-to-interferenceplus-noise ratio (SINR) for UE $k$ reads as

$\operatorname{SINR}_{k} \triangleq \frac{\left|\sum_{b \in \mathcal{B}} \mathbf{v}_{b, k}^{\mathrm{H}} \mathbf{H}_{b, k} \mathbf{w}_{k}\right|^{2}}{\sum_{\bar{k} \in \mathcal{K} \backslash\{k\}}\left|\sum_{b \in \mathcal{B}} \mathbf{v}_{b, k}^{\mathrm{H}} \mathbf{H}_{b, \bar{k}} \mathbf{w}_{\bar{k}}\right|^{2}+\sigma_{\mathrm{BS}}^{2} \sum_{b \in \mathcal{B}}\left\|\mathbf{v}_{b, k}\right\|^{2}}$.

Finally, the sum rate (measured in $\mathrm{bps} / \mathrm{Hz}$ ) is given by

$$
R \triangleq \sum_{k \in \mathcal{K}} \log _{2}\left(1+\mathrm{SINR}_{k}\right)
$$

This paper proposes a distributed joint receiver design where each BS $b$ optimizes its BS-specific combining vectors $\left\{\mathbf{v}_{b, k}\right\}_{k \in \mathcal{K}}$ locally while coordinating with the other BSs. For ease of comparison, we also illustrate the centralized joint receiver design, where the aggregated combining vectors $\left\{\mathbf{v}_{k}\right\}_{k \in \mathcal{K}}$ are optimized by the CPU and the BS-specific combining vectors are fed back to the BSs. In both cases, the precoding vectors $\left\{\mathbf{w}_{k}\right\}_{k \in \mathcal{K}}$ are computed locally and independently by the corresponding UEs. Next, we describe realistic pilot-aided CSI acquisition at both the BSs and the UEs, which will be needed in Sections IV and V.

\section{A. Uplink Pilot-Aided Channel Estimation}

Let $\mathbf{h}_{b, k} \triangleq \mathbf{H}_{b, k} \mathbf{w}_{k} \in \mathbb{C}^{M \times 1}$ denote the effective uplink channel vector between UE $k$ and BS $b$, and let $\mathbf{p}_{k} \in \mathbb{C}^{\tau \times 1}$ denote the pilot assigned to UE $k$, with $\left\|\mathbf{p}_{k}\right\|^{2}=\tau$. In the uplink pilot-aided channel estimation phase, each UE $k$ synchronously ${ }^{1}$ transmits its pilot $\mathbf{p}_{k}$ precoded with $\mathbf{w}_{k}$, i.e.,

$$
\mathbf{X}_{k}^{\mathrm{UL}-1} \triangleq \mathbf{w}_{k} \mathbf{p}_{k}^{\mathrm{H}} \in \mathbb{C}^{N \times \tau} .
$$

Then, the receive signal at $\mathrm{BS} b$ is given by

$$
\begin{aligned}
\mathbf{Y}_{b}^{\mathrm{UL}-1} & \triangleq \sum_{k \in \mathcal{K}} \mathbf{H}_{b, k} \mathbf{X}_{k}^{\mathrm{UL}-1}+\mathbf{Z}_{b}^{\mathrm{UL}-1} \\
& =\sum_{k \in \mathcal{K}} \mathbf{h}_{b, k} \mathbf{p}_{k}^{\mathrm{H}}+\mathbf{Z}_{b}^{\mathrm{UL}-1} \in \mathbb{C}^{M \times \tau}
\end{aligned}
$$

where $\mathbf{Z}_{b}^{\mathrm{UL}-1} \in \mathbb{C}^{M \times \tau}$ is the AWGN term at BS $b$ with elements distributed as $\mathcal{C N}\left(0, \sigma_{\mathrm{BS}}^{2}\right)$, and the least-squares estimate of $\mathbf{h}_{b, k}$ is obtained as

$$
\begin{aligned}
\hat{\mathbf{h}}_{b, k} & \triangleq \frac{1}{\tau} \mathbf{Y}_{b}^{\mathrm{UL}-1} \mathbf{p}_{k} \\
& =\mathbf{h}_{b, k}+\frac{1}{\tau} \sum_{\bar{k} \in \mathcal{K} \backslash\{k\}} \mathbf{h}_{b, \bar{k}} \mathbf{p}_{\bar{k}}^{\mathrm{H}} \mathbf{p}_{k}+\frac{1}{\tau} \mathbf{Z}_{b}^{\mathrm{UL}-1} \mathbf{p}_{k} .
\end{aligned}
$$

Here, perfect channel estimation is attained when:

i) The pilot contamination in the second term of (9) is eliminated using, e.g., orthogonal pilots (i.e., $\left\{\mathbf{p}_{\bar{k}}^{\mathrm{H}} \mathbf{p}_{k}=\right.$

\footnotetext{
${ }^{1}$ The UE- and BS-specific synchronization issues are discussed in [2], [7].
}

$0\}_{\bar{k} \in \mathcal{K} \backslash\{k\}}$ ) or non-orthogonal random pilots with infinite length (i.e., $\tau \rightarrow \infty$ );

ii) The channel estimation noise in the third term of (9) is eliminated using pilots with infinite length.

These observations also hold for (14) and (19) in the following.

In the centralized joint receiver design, the estimation of the channel matrix $\mathbf{H}_{b, k}$ calls for $N$ antenna-specific pilots for UE $k$. In this context, let $\mathbf{P}_{k} \in \mathbb{C}^{\tau \times N}$ denote the pilot matrix assigned to UE $k$, with $\left\|\mathbf{P}_{k}\right\|_{\mathrm{F}}^{2}=\tau N$. As in (5), each UE $k$ synchronously transmits its pilot matrix, i.e.,

$$
\mathbf{X}_{k}^{\mathrm{UL}} \triangleq \sqrt{\beta^{\mathrm{u}}} \mathbf{P}_{k}^{\mathrm{H}} \in \mathbb{C}^{N \times \tau}
$$

where the scaling factor $\beta^{\mathrm{UL}} \triangleq \rho_{\mathrm{UE}} / N$ ensures that each column of $\mathbf{X}_{k}^{\mathrm{UL}}$ complies with the UE transmit power constraint. Then, the receive signal at BS $b$ is given by

$$
\begin{aligned}
\mathbf{Y}_{b}^{\mathrm{UL}} & \triangleq \sum_{k \in \mathcal{K}} \mathbf{H}_{b, k} \mathbf{X}_{k}^{\mathrm{UL}}+\mathbf{Z}_{b}^{\mathrm{UL}} \\
& =\sqrt{\beta^{\mathrm{UL}}} \sum_{k \in \mathcal{K}} \mathbf{H}_{b, k} \mathbf{P}_{k}^{\mathrm{H}}+\mathbf{Z}_{b}^{\mathrm{UL}} \in \mathbb{C}^{M \times \tau}
\end{aligned}
$$

where $\mathbf{Z}_{b}^{\mathrm{UL}} \in \mathbb{C}^{M \times \tau}$ is the AWGN term at BS $b$ with elements distributed as $\mathcal{C N}\left(0, \sigma_{\mathrm{BS}}^{2}\right)$, and the least-squares estimate of $\mathbf{H}_{b, k}$ is obtained as

$$
\begin{aligned}
\hat{\mathbf{H}}_{b, k} & \triangleq \frac{1}{\tau \sqrt{\beta^{\mathrm{UL}}}} \mathbf{Y}_{b}^{\mathrm{UL}} \mathbf{P}_{k} \\
& =\frac{1}{\tau} \sum_{\bar{k} \in \mathcal{K}} \mathbf{H}_{b, \bar{k}} \mathbf{P}_{\bar{k}}^{\mathrm{H}} \mathbf{P}_{k}+\frac{1}{\tau \sqrt{\beta^{\mathrm{UL}}}} \mathbf{Z}_{b}^{\mathrm{UL}-1} \mathbf{P}_{k} .
\end{aligned}
$$

\section{B. Downlink Pilot-Aided Channel Estimation}

Let $\mathbf{g}_{k} \triangleq \sum_{b \in \mathcal{B}} \mathbf{H}_{b, k}^{\mathrm{H}} \mathbf{v}_{b, k} \in \mathbb{C}^{N \times 1}$ denote the effective downlink channel vector between all the BSs and UE $k$. In the downlink pilot-aided channel estimation phase, each BS $b$ synchronously transmits a superposition of the pilots $\left\{\mathbf{p}_{k}\right\}_{k \in \mathcal{K}}$ each precoded with the corresponding combining vector $\mathbf{v}_{b, k}$, i.e.,

$$
\mathbf{X}_{b}^{\mathrm{DL}} \triangleq \sqrt{\beta^{\mathrm{DL}}} \sum_{k \in \mathcal{K}} \mathbf{v}_{b, k} \mathbf{p}_{k}^{\mathrm{H}} \in \mathbb{C}^{M \times \tau}
$$

where the scaling factor $\beta^{\mathrm{DL}}$ (equal for all the BSs) ensures that each column of $\mathbf{X}_{b}^{\mathrm{DL}}$ complies with the BS transmit power constraint. Then, the receive signal at UE $k$ is given by

$$
\begin{aligned}
\mathbf{Y}_{k}^{\mathrm{DL}} & \triangleq \sum_{b \in \mathcal{B}} \mathbf{H}_{b, k}^{\mathrm{H}} \mathbf{X}_{b}^{\mathrm{DL}}+\mathbf{Z}_{k}^{\mathrm{DL}} \\
& =\sqrt{\beta^{\mathrm{DL}}} \sum_{b \in \mathcal{B}} \sum_{\bar{k} \in \mathcal{K}} \mathbf{H}_{b, k}^{\mathrm{H}} \mathbf{v}_{b, \bar{k}} \mathbf{p}_{\bar{k}}^{\mathrm{H}}+\mathbf{Z}_{k}^{\mathrm{DL}} \in \mathbb{C}^{N \times \tau}
\end{aligned}
$$

where $\mathbf{Z}_{k}^{\mathrm{DL}} \in \mathbb{C}^{N \times \tau}$ is the AWGN term at UE $k$ with elements distributed as $\mathcal{C N}\left(0, \sigma_{\mathrm{uE}}^{2}\right)$, and the least-squares estimate of $\mathbf{g}_{k}$ is obtained as

$$
\begin{aligned}
\hat{\mathbf{g}}_{k} & \triangleq \frac{1}{\tau \sqrt{\beta^{\mathrm{DL}}}} \mathbf{Y}_{k}^{\mathrm{DL}} \mathbf{p}_{k} \\
& =\mathbf{g}_{k}+\frac{1}{\tau} \sum_{b \in \mathcal{B}} \sum_{\bar{k} \in \mathcal{K} \backslash\{k\}} \mathbf{H}_{b, k}^{\mathrm{H}} \mathbf{v}_{b, \bar{k}} \mathbf{p}_{\bar{k}}^{\mathrm{H}} \mathbf{p}_{k}+\frac{1}{\tau \sqrt{\beta^{\mathrm{DL}}}} \mathbf{Z}_{k}^{\mathrm{DL}} \mathbf{p}_{k} .
\end{aligned}
$$

\section{Problem Formulation with Perfect CSI}

As in our previous work [9], which focused on the downlink scenario, the BS-specific combining vectors $\left\{\mathbf{v}_{b, k}\right\}_{b \in \mathcal{B}, k \in \mathcal{K}}$ 
and the precoding vectors $\left\{\mathbf{w}_{k}\right\}_{k \in \mathcal{K}}$ are optimized by solving the sum mean squared error (MSE) minimization problem. In doing so, it is useful to first illustrate the case of perfect CSI before considering realistic pilot-aided CSI acquisition at both the BSs and the UEs in Section IV.

From (2), we define the MSE at UE $k$ as

$$
\begin{aligned}
\mathrm{MSE}_{k} \triangleq & \mathbb{E}\left[\left|\sum_{b \in \mathcal{B}} \mathbf{v}_{b, k}^{\mathrm{H}} \mathbf{y}_{b}-d_{k}\right|^{2}\right] \\
= & \sum_{\bar{k} \in \mathcal{K}}\left|\sum_{b \in \mathcal{B}} \mathbf{v}_{b, k}^{\mathrm{H}} \mathbf{H}_{b, \bar{k}} \mathbf{w}_{\bar{k}}\right|^{2}-2 \operatorname{Re}\left[\sum_{b \in \mathcal{B}} \mathbf{v}_{b, k}^{\mathrm{H}} \mathbf{H}_{b, k} \mathbf{w}_{k}\right] \\
& +\sigma_{\mathrm{BS}}^{2} \sum_{b \in \mathcal{B}}\left\|\mathbf{v}_{b, k}\right\|^{2}+1
\end{aligned}
$$

which is convex with respect to either the transmit or the receive strategies (but not jointly convex with respect to both). Therefore, we use alternate optimization, i.e., we optimize the combining vectors for fixed precoding vectors and vice versa in an iterative best-response fashion [10].

Optimization of the precoding vectors. The precoding vectors $\left\{\mathbf{w}_{k}\right\}_{k \in \mathcal{K}}$ are computed locally and independently by the UEs such that each UE $k$ minimizes $\mathrm{MSE}_{k}$ in (21). From the perspective of UE $k$, we can rewrite the MSE as

$$
\mathrm{MSE}_{k}=\mathbf{w}_{k}^{\mathrm{H}} \mathbf{\Psi}_{k} \mathbf{w}_{k}-2 \operatorname{Re}\left[\mathbf{g}_{k}^{\mathrm{H}} \mathbf{w}_{k}\right]+\sigma_{\mathrm{BS}}^{2} \sum_{b \in \mathcal{B}}\left\|\mathbf{v}_{b, k}\right\|^{2}+1
$$

where we have defined

$$
\mathbf{\Psi}_{k} \triangleq \sum_{\bar{k} \in \mathcal{K}}\left(\sum_{b \in \mathcal{B}} \mathbf{H}_{b, k}^{\mathrm{H}} \mathbf{v}_{b, \bar{k}}\right)\left(\sum_{b \in \mathcal{B}} \mathbf{v}_{b, \bar{k}}^{\mathrm{H}} \mathbf{H}_{b, k}\right) .
$$

The precoding vector $\mathbf{w}_{k}$ that minimizes (22) is the MMSE precoder

$$
\mathbf{w}_{k}=\left(\mathbf{\Psi}_{k}+\lambda_{k} \mathbf{I}_{N}\right)^{-1} \mathbf{g}_{k}
$$

where $\lambda_{k}$ is the dual variable corresponding to the transmit power constraint of UE $k$, which can be efficiently optimized via bisection methods. Note that $\mathbf{w}_{k}$ can be computed locally by UE $k$ as in (24) if $\boldsymbol{\Psi}_{k}$ in (23) and the effective downlink channel $\mathbf{g}_{k}$ are known by UE $k$.

Optimization of the combining vectors. The BS-specific combining vectors $\left\{\mathbf{v}_{b, k}\right\}_{b \in \mathcal{B}, k \in \mathcal{K}}$ are computed as the solutions of the sum MSE minimization problem. To this end, we introduce the following preliminary definitions: $\mathbf{h}_{k} \triangleq$ $\left[\mathbf{h}_{1, k}^{\mathrm{T}}, \ldots, \mathbf{h}_{B, k}^{\mathrm{T}}\right]^{\mathrm{T}} \in \mathbb{C}^{B M \times 1}, \mathbf{H} \triangleq\left[\mathbf{h}_{1}, \ldots, \mathbf{h}_{K}\right] \in \mathbb{C}^{B M \times K}$, $\mathbf{V} \triangleq\left[\mathbf{v}_{1}, \ldots, \mathbf{v}_{K}\right] \in \mathbb{C}^{B M \times K}, \mathbf{\Phi} \triangleq \sum_{k \in \mathcal{K}} \mathbf{h}_{k} \mathbf{h}_{k}^{\mathrm{H}} \in$ $\mathbb{C}^{B M \times B M}$, where the latter may be rewritten as

$$
\boldsymbol{\Phi} \triangleq\left[\begin{array}{ccc}
\boldsymbol{\Phi}_{11} & \ldots & \boldsymbol{\Phi}_{1 B} \\
\vdots & \ddots & \vdots \\
\boldsymbol{\Phi}_{1 B}^{\mathrm{H}} & \ldots & \boldsymbol{\Phi}_{B B}
\end{array}\right]
$$

with $\boldsymbol{\Phi}_{b \bar{b}} \triangleq \sum_{k \in \mathcal{K}} \mathbf{h}_{b, k} \mathbf{h}_{\bar{b}, k}^{\mathrm{H}} \in \mathbb{C}^{M \times M}$. Finally, the sum MSE can be expressed as

$\sum_{k \in \mathcal{K}} \operatorname{MSE}_{k}=\operatorname{tr}\left(\mathbf{V}^{\mathrm{H}}\left(\mathbf{\Phi}+\sigma_{\mathrm{BS}}^{2} \mathbf{I}_{B M}\right) \mathbf{V}\right)-2 \operatorname{Re}\left[\operatorname{tr}\left(\mathbf{V}^{\mathrm{H}} \mathbf{H}\right)\right]+K$.

We now describe the centralized and the distributed joint receiver designs with perfect CSI.

\section{A. Centralized Joint Receiver Design}

In the centralized joint receiver design, the alternate optimization between the combining and the precoding vectors is carried out transparently at the CPU. For fixed precoding vectors, the CPU solves the sum MSE minimization problem

$$
\min _{\left\{\mathbf{v}_{k}\right\}_{k \in \mathcal{K}}} \operatorname{tr}\left(\mathbf{V}^{\mathrm{H}}\left(\mathbf{\Phi}+\sigma_{\mathrm{BS}}^{2} \mathbf{I}_{B M}\right) \mathbf{V}\right)-2 \operatorname{Re}\left[\operatorname{tr}\left(\mathbf{V}^{\mathrm{H}} \mathbf{H}\right)\right]
$$

and, for each UE $k$, the first-order optimality condition of (27) yields the well-known centralized MMSE receiver

$$
\mathbf{v}_{k}=\left(\mathbf{\Phi}+\sigma_{\mathrm{BS}}^{2} \mathbf{I}_{B M}\right)^{-1} \mathbf{h}_{k} .
$$

First, each BS $b$ acquires the channel matrices $\left\{\mathbf{H}_{b, k}\right\}_{k \in \mathcal{K}}$ and forwards them to the CPU via backhaul signaling. Then, the CPU computes the aggregated combining vectors $\left\{\mathbf{v}_{k}\right\}_{k \in \mathcal{K}}$ as in (28) together with the precoding vectors $\left\{\mathbf{w}_{k}\right\}_{k \in \mathcal{K}}$ as in (24) by means of alternate optimization. After this stage, the CPU feeds back the BS-specific combining vectors $\left\{\mathbf{v}_{b, k}\right\}_{k \in \mathcal{K}}$ to each BS $b$ via backhaul signaling. Lastly, each UE $k$ acquires $\boldsymbol{\Psi}_{k}$ in (23) and the effective downlink channel $\mathbf{g}_{k}$, based on which it computes its precoding vector $\mathbf{w}_{k}$ as in (24).

\section{B. Distributed Joint Receiver Design}

In the distributed joint receiver design, the alternate optimization of the combining and the precoding vectors is carried out by means of iterative bi-directional signaling between the BSs and the UEs (see [8], [11]). For fixed precoding vectors, the BSs solve the sum MSE minimization problem

$$
\min _{\left\{\mathbf{v}_{b, k}\right\}_{b \in \mathcal{B}, k \in \mathcal{K}}} \operatorname{tr}\left(\mathbf{V}^{\mathrm{H}}\left(\mathbf{\Phi}+\sigma_{\mathrm{BS}}^{2} \mathbf{I}_{B M}\right) \mathbf{V}\right)-2 \operatorname{Re}\left[\operatorname{tr}\left(\mathbf{V}^{\mathrm{H}} \mathbf{H}\right)\right]
$$

and, for each BS $b$ and for each UE $k$, the first-order optimality condition of (29) yields the distributed joint receiver solution

$$
\mathbf{v}_{b, k}=\left(\boldsymbol{\Phi}_{b b}+\sigma_{\mathrm{BS}}^{2} \mathbf{I}_{M}\right)^{-1}\left(\mathbf{h}_{b, k}-\boldsymbol{\xi}_{b, k}\right)
$$

where we have defined

$$
\boldsymbol{\xi}_{b, k} \triangleq \sum_{\bar{b} \in \mathcal{B} \backslash\{b\}} \boldsymbol{\Phi}_{b \bar{b}} \mathbf{v}_{\bar{b}, k} .
$$

As detailed in [9], $\boldsymbol{\xi}_{b, k}$ in (31) incorporates the missing cross-terms containing implicit information about the channel correlation between BS $b$ and the other BSs and about the combining vectors adopted by the latter for UE $k$. In this respect, omitting $\boldsymbol{\xi}_{b, k}$ from (30) results in the highly suboptimal local MMSE receiver. Observe that, while the effective uplink channels $\left\{\mathbf{h}_{b, k}\right\}_{k \in \mathcal{K}}$ can be acquired locally by each BS $b$, the acquisition of $\left\{\boldsymbol{\xi}_{b, k}\right\}_{k \in \mathcal{K}}$ still requires CSI exchange among the BSs via backhaul signaling [8]. In Section V, we describe a practical method to implement the distributed joint receiver design that relies solely on more flexible OTA signaling.

According to the parallel optimization framework [12], the distributed joint receiver design can be realized in an iterative best-response fashion. For a given UE $k$, at each iteration $i$, each BS $b$ locally computes $\mathbf{v}_{b, k}$ as in (30) for fixed $\left\{\mathbf{v}_{\bar{b}, k}\right\}_{\bar{b} \in \mathcal{B} \backslash\{b\}}$ in parallel with the other BSs; then, each BS $b$ updates its combining vector as

$$
\mathbf{v}_{b, k}^{(i)}=(1-\alpha) \mathbf{v}_{b, k}^{(i-1)}+\alpha \mathbf{v}_{b, k}
$$

where the step size $\alpha \in(0,1]$ defines a tradeoff between con- 
vergence speed and accuracy [8], [12]. Hence, the distributed joint receiver design is implemented as follows. First, for fixed precoding vectors $\left\{\mathbf{w}_{k}\right\}_{k \in \mathcal{K}}$, each BS $b$ acquires the effective uplink channels $\left\{\mathbf{h}_{b, k}\right\}_{k \in \mathcal{K}}$ and the vectors $\left\{\boldsymbol{\xi}_{b, k}\right\}_{k \in \mathcal{K}}$. Then, it computes its BS-specific combining vectors $\left\{\mathbf{v}_{b, k}\right\}_{k \in \mathcal{K}}$ locally as in (30) and it updates them as in (32). Subsequently, each UE $k$ acquires $\boldsymbol{\Psi}_{k}$ in (23) and the effective downlink channel $\mathbf{g}_{k}$, based on which it computes its precoding vector $\mathbf{w}_{k}$ locally as in (24). This procedure is iterated until a predefined termination criterion is satisfied.

\section{Problem Formulation with Imperfect CSI}

In this section, we reformulate the problem of Section III under realistic pilot-aided CSI acquisition at both the BSs and the UEs (see Sections II-A and II-B). In doing so, we first describe the centralized joint receiver design in Section IV-A and then focus on its distributed counterpart in Section IV-B.

\section{A. Centralized Joint Receive Design}

In the centralized joint receiver design, the CPU computes the precoding vectors and the aggregated combining vectors for each UE $k$ as

$$
\begin{aligned}
\mathbf{w}_{k}= & \left(\sum_{\bar{k} \in \mathcal{K}}\left(\sum_{b \in \mathcal{B}} \hat{\mathbf{H}}_{b, k}^{\mathrm{H}} \mathbf{v}_{b, \bar{k}}\right)\left(\sum_{b \in \mathcal{B}} \mathbf{v}_{b, \bar{k}}^{\mathrm{H}} \hat{\mathbf{H}}_{b, k}\right)+\lambda_{k}^{2} \mathbf{I}_{N}\right)^{-1} \\
& \times \sum_{b \in \mathcal{B}} \hat{\mathbf{H}}_{b, k}^{\mathrm{H}} \mathbf{v}_{b, k} \\
\mathbf{v}_{k}= & \left(\sum_{\bar{k} \in \mathcal{K}} \hat{\mathbf{H}}_{\bar{k}} \mathbf{w}_{\bar{k}} \mathbf{w}_{\bar{k}}^{\mathrm{H}} \hat{\mathbf{H}}_{\bar{k}}^{\mathrm{H}}+\sigma_{\mathrm{BS}}^{2} \mathbf{I}_{B M}\right)^{-1} \hat{\mathbf{H}}_{k} \mathbf{w}_{k}
\end{aligned}
$$

respectively. Here, (33) and (34) are obtained from minimizing $\mathrm{MSE}_{k}$ in (21) after replacing the channels $\left\{\mathbf{H}_{b, k}\right\}_{b \in \mathcal{B}}$ with the estimated channels $\left\{\hat{\mathbf{H}}_{b, k}\right\}_{b \in \mathcal{B}}$ (obtained as in (14)), and are equal to (24) and (28), respectively, for perfect CSI. Note that such scheme is very sensitive to imperfect channel estimation as it relies on a single pilot-aided CSI acquisition (see [9] for more details).

\section{B. Distributed Joint Receiver Design}

For the computation of the precoding vectors $\left\{\mathbf{w}_{k}\right\}_{k \in \mathcal{K}}$, upon receiving $\mathbf{Y}_{k}^{\mathrm{DL}}$ in (17), each UE $k$ obtains

$$
\begin{aligned}
\frac{1}{\tau \beta^{\mathrm{DL}}} \mathbf{Y}_{k}^{\mathrm{DL}}\left(\mathbf{Y}_{k}^{\mathrm{DL}}\right)^{\mathrm{H}}=\mathbf{\Psi}_{k} & +\frac{1}{\tau} \sum_{\substack{\bar{k}, j \in \mathcal{K} \\
\bar{k} \neq j}}\left(\sum_{b \in \mathcal{B}} \mathbf{H}_{b, k}^{\mathrm{H}} \mathbf{v}_{b, \bar{k}}\right) \\
& \times\left(\sum_{b \in \mathcal{B}} \mathbf{v}_{b, j}^{\mathrm{H}} \mathbf{H}_{b, k}\right) \mathbf{p}_{\bar{k}}^{\mathrm{H}} \mathbf{p}_{j}+\mathbf{N}_{k}^{\mathrm{DL}}
\end{aligned}
$$

with $\Psi_{k}$ defined in (23), and where we have defined

$$
\begin{aligned}
\mathbf{N}_{k}^{\mathrm{DL}} \triangleq \frac{1}{\tau}\left(\frac { 1 } { \sqrt { \beta ^ { \mathrm { DL } } } } \sum _ { b \in \mathcal { B } } \sum _ { \overline { k } \in \mathcal { K } } \left(\mathbf{H}_{b, k}^{\mathrm{H}} \mathbf{v}_{b, \bar{k}} \mathbf{p}_{\bar{k}}^{\mathrm{H}}\left(\mathbf{Z}_{k}^{\mathrm{DL}}\right)^{\mathrm{H}}\right.\right. \\
\left.\left.+\mathbf{Z}_{k}^{\mathrm{DL}} \mathbf{p}_{\bar{k}} \mathbf{v}_{b, \bar{k}}^{\mathrm{H}} \mathbf{H}_{b, k}\right)+\frac{1}{\beta^{\mathrm{DL}}} \mathbf{Z}_{k}^{\mathrm{DL}}\left(\mathbf{Z}_{k}^{\mathrm{DL}}\right)^{\mathrm{H}}\right) .
\end{aligned}
$$

In presence of perfect channel estimation, we would have that:

i) The pilot contamination in the second term of (35) is eliminated; ii) As $\tau \rightarrow \infty$, the first term of (36) is eliminated and $\frac{1}{\tau \beta^{\mathrm{DL}}} \mathbf{Z}_{k}^{\mathrm{DL}}\left(\mathbf{Z}_{k}^{\mathrm{DL}}\right)^{\mathrm{H}} \rightarrow \frac{\sigma_{\mathrm{UE}}^{2}}{\beta^{\mathrm{DL}}} \mathbf{I}_{N}$.

Thus, (35) can be used as an estimate of $\Psi_{k}+\frac{\sigma_{\mathrm{UE}}^{2}}{\beta \mathrm{LE}} \mathbf{I}_{N}$ and, consequently, we can obtain an estimate of $\mathrm{MSE}_{k}$ in (22) as

$$
\begin{aligned}
\mathrm{MSE}_{k} \simeq & \frac{1}{\tau \beta^{\mathrm{DL}}} \mathbf{w}_{k}^{\mathrm{H}}\left(\mathbf{Y}_{k}^{\mathrm{DL}}\left(\mathbf{Y}_{k}^{\mathrm{DL}}\right)^{\mathrm{H}}-\tau \sigma_{\mathrm{UE}}^{2} \mathbf{I}_{N}\right) \mathbf{w}_{k} \\
& -\frac{2}{\tau \sqrt{\beta^{\mathrm{DL}}}} \operatorname{Re}\left[\mathbf{w}_{k}^{\mathrm{H}} \mathbf{Y}_{k}^{\mathrm{DL}} \mathbf{p}_{k}\right]+\sigma_{\mathrm{BS}}^{2} \sum_{b \in \mathcal{B}}\left\|\mathbf{v}_{b, k}\right\|^{2}+1
\end{aligned}
$$

Finally, the precoding vector $\mathbf{w}_{k}$ can be computed locally by UE $k$ as

$$
\mathbf{w}_{k}=\sqrt{\beta^{\mathrm{DL}}}\left(\mathbf{Y}_{k}^{\mathrm{DL}}\left(\mathbf{Y}_{k}^{\mathrm{DL}}\right)^{\mathrm{H}}+\tau\left(\lambda_{k}-\sigma_{\mathrm{UE}}^{2}\right) \mathbf{I}_{N}\right)^{-1} \mathbf{Y}_{k}^{\mathrm{DL}} \mathbf{p}_{k}
$$

which is equal to (24) for perfect CSI.

For the computation of the combining vectors $\left\{\mathbf{v}_{b, k}\right\}_{b \in \mathcal{B}, k \in \mathcal{K}}$, let us define $\mathbf{Y}^{\mathrm{UL}-1} \triangleq$ $\left[\left(\mathbf{Y}_{1}^{\mathrm{UL}-1}\right)^{\mathrm{T}}, \ldots,\left(\mathbf{Y}_{K}^{\mathrm{UL}-1}\right)^{\mathrm{T}}\right]^{\mathrm{T}} \in \mathbb{C}^{B M \times \tau}$ and $\mathbf{P} \triangleq\left[\mathbf{p}_{1}, \ldots, \mathbf{p}_{K}\right] \in$ $\mathbb{C}^{\tau \times K}$. From (7), for each BS pair $b$ and $\bar{b}$, we have

$$
\frac{1}{\tau} \mathbf{Y}_{b}^{\mathrm{UL}-1}\left(\mathbf{Y}_{\bar{b}}^{\mathrm{UL}-1}\right)^{\mathrm{H}}=\boldsymbol{\Phi}_{b \bar{b}}+\frac{1}{\tau} \sum_{\substack{k, \bar{k} \in \mathcal{K} \\ k \neq \bar{k}}} \mathbf{h}_{b, k} \mathbf{h}_{\bar{b}, \bar{k}}^{\mathrm{H}} \mathbf{p}_{k}^{\mathrm{H}} \mathbf{p}_{\bar{k}}+\mathbf{N}_{b \bar{b}}^{\mathrm{UL}-1}
$$

where we have defined

$$
\begin{aligned}
\mathbf{N}_{b \bar{b}}^{\mathrm{UL}-1} \triangleq \frac{1}{\tau}\left(\sum _ { k \in \mathcal { K } } \left(\mathbf{h}_{b, k} \mathbf{p}_{k}^{\mathrm{H}}\left(\mathbf{Z}_{\bar{b}}^{\mathrm{UL}-1}\right)^{\mathrm{H}}+\right.\right. & \left.\mathbf{Z}_{b}^{\mathrm{UL}-1} \mathbf{p}_{k} \mathbf{h}_{\bar{b}, k}^{\mathrm{H}}\right) \\
& \left.+\mathbf{Z}_{b}^{\mathrm{UL}-1}\left(\mathbf{Z}_{\bar{b}}^{\mathrm{UL}-1}\right)^{\mathrm{H}}\right) .
\end{aligned}
$$

In presence of perfect channel estimation, we would have that:

i) The pilot contamination in the second term of (39) is eliminated;

ii) As $\tau \rightarrow \infty$, the first term of (40) is eliminated, whereas $\frac{1}{\tau} \mathbf{Z}_{b}^{\mathrm{UL}-1}\left(\mathbf{Z}_{\bar{b}}^{\mathrm{UL}-1}\right)^{\mathrm{H}} \rightarrow \mathbf{0}$ if $\bar{b} \neq b$ and $\frac{1}{\tau} \mathbf{Z}_{b}^{\mathrm{UL}-1}\left(\mathbf{Z}_{b}^{\mathrm{UL}-1}\right)^{\mathrm{H}} \rightarrow \sigma_{\mathrm{BS}}^{2} \mathbf{I}_{M}$. Thus, (39) can be used as an estimate of $\boldsymbol{\Phi}_{b \bar{b}}$ if $\bar{b} \neq b$ or of $\boldsymbol{\Phi}_{b b}+\sigma_{\mathrm{BS}}^{2} \mathbf{I}_{M}$ if $\bar{b}=b$ and, consequently, $\frac{1}{\tau} \mathbf{Y}^{\mathrm{UL}-1}\left(\mathbf{Y}^{\mathrm{UL}-1}\right)^{\mathrm{H}}$ can be used as an estimate of $\boldsymbol{\Phi}+\sigma_{\mathrm{BS}}^{2} \mathbf{I}_{B M}$. Building upon this, we can write the estimated sum MSE as

$$
\begin{aligned}
\sum_{k \in \mathcal{K}} \mathrm{MSE}_{k} \simeq \frac{1}{\tau} \operatorname{tr}\left(\mathbf{V}^{\mathrm{H}} \mathbf{Y}^{\mathrm{UL}-1}\left(\mathbf{Y}^{\mathrm{UL}-1}\right)^{\mathrm{H}} \mathbf{V}\right) \\
-\frac{2}{\tau} \operatorname{Re}\left[\operatorname{tr}\left(\mathbf{V}^{\mathrm{H}} \mathbf{Y}^{\mathrm{UL}-1} \mathbf{P}\right)\right]+K .
\end{aligned}
$$

Finally, for each BS $b$ and for each UE $k$, the distributed joint receiver solution is given by

$$
\mathbf{v}_{b, k}=\left(\mathbf{Y}_{b}^{\mathrm{UL}-1}\left(\mathbf{Y}_{b}^{\mathrm{UL}-1}\right)^{\mathrm{H}}\right)^{-1} \mathbf{Y}_{b}^{\mathrm{UL}-1}\left(\mathbf{p}_{k}-\sum_{\bar{b} \in \mathcal{B} \backslash\{b\}}\left(\mathbf{Y}_{\bar{b}}^{\mathrm{UL}-1}\right)^{\mathrm{H}} \mathbf{v}_{\bar{b}, k}\right)
$$

which is equal to (30) for perfect CSI. Here, each BS $b$ needs to acquire $\left(\mathbf{Y}_{\bar{b}}^{\mathrm{UL}-1}\right)^{\mathrm{H}} \mathbf{v}_{\bar{b}, k} \in \mathbb{C}^{\tau \times 1}$ from each BS $\bar{b} \neq b$ via backhaul signaling as illustrated in [8]. The amount of such signaling scales not only with the pilot length $\tau$ but also with the number of BSs $B$ and the number of UEs $K$; furthermore, the backhaul introduces delays in the CSI exchange among the BSs leading to further performance losses. In the next section, we describe a practical method to implement the distributed joint receiver design via OTA signaling. 


$$
\begin{aligned}
\frac{1}{\tau}\left(\frac{1}{\sqrt{\beta^{\mathrm{UL}-2} \beta^{\mathrm{DL}}}} \mathbf{Y}_{b}^{\mathrm{UL}-2} \mathbf{p}_{k}-\left(\mathbf{Y}_{b}^{\mathrm{UL}-1}\left(\mathbf{Y}_{b}^{\mathrm{UL}-1}\right)^{\mathrm{H}}-\tau \sigma_{\mathrm{BS}}^{2} \mathbf{I}_{M}\right) \mathbf{v}_{b, k}\right) \\
=\boldsymbol{\xi}_{b, k}+\frac{1}{\tau}\left(\sum_{\bar{k} \in \mathcal{K} \backslash\{k\}} \sum_{\bar{b} \in \mathcal{B}} \boldsymbol{\Phi}_{b \bar{b}} \mathbf{v}_{\bar{b}, \bar{k}} \mathbf{p}_{\bar{k}}^{\mathrm{H}} \mathbf{p}_{k}-\sum_{\substack{\bar{k}, j \in \mathcal{K} \\
\bar{k} \neq j}} \mathbf{h}_{b, \bar{k}} \mathbf{h}_{b, j}^{\mathrm{H}} \mathbf{v}_{b, k} \mathbf{p}_{\bar{k}}^{\mathrm{H}} \mathbf{p}_{j}\right)+\mathbf{n}_{b, k}^{\mathrm{UL}-2}+\left(\sigma_{\mathrm{BS}}^{2} \mathbf{I}_{M}-\mathbf{N}_{b b}^{\mathrm{UL}-1}\right) \mathbf{v}_{b, k}
\end{aligned}
$$

\section{Distributed Joint Receiver Design Via OTA SIGNALING}

Building upon the OTA signaling framework proposed in [9] for the downlink scenario, each BS $b$ can obtain an estimate of $\boldsymbol{\xi}_{b, k}$ in (31) without any backhaul signaling for CSI exchange among the BSs. To this end, we introduce an additional uplink signaling resource, whereby each UE $k$ synchronously transmits $\mathbf{Y}_{k}^{\mathrm{DL}}$ in (17) precoded with the rank-1 matrix $\mathbf{w}_{k} \mathbf{w}_{k}^{\mathrm{H}}$, i.e.,

$$
\mathbf{X}_{k}^{\mathrm{UL}-2} \triangleq \sqrt{\beta^{\mathrm{UL}-2}} \mathbf{w}_{k} \mathbf{w}_{k}^{\mathrm{H}} \mathbf{Y}_{k}^{\mathrm{DL}} \in \mathbb{C}^{N \times \tau}
$$

where the scaling factor $\beta^{\text {UL-2 }}$ (equal for all the UEs) ensures that each column of $\mathbf{X}_{k}^{\mathrm{UL}-2}$ is transmitted with a large enough power while still complying with the UE transmit power constraint. More specifically, each UE $k$ uses its precoding vector $\mathbf{w}_{k}$ to combine $\mathbf{Y}_{k}^{\mathrm{DL}}$ and then transmits it using again $\mathbf{w}_{k}$. Then, the receive signal at BS $b$ is given by

$$
\begin{aligned}
\mathbf{Y}_{b}^{\mathrm{UL}-2} \triangleq & \sum_{k \in \mathcal{K}} \mathbf{H}_{b, k} \mathbf{X}_{k}^{\mathrm{UL}-2}+\mathbf{Z}_{b}^{\mathrm{UL}-2} \\
= & \sqrt{\beta^{\mathrm{UL}-2}} \sum_{k \in \mathcal{K}} \mathbf{h}_{b, k} \mathbf{w}_{k}^{\mathrm{H}}\left(\sqrt{\beta^{\mathrm{DL}}} \sum_{\bar{b} \in \mathcal{B}} \sum_{\bar{k} \in \mathcal{K}} \mathbf{H}_{\bar{b}, k}^{\mathrm{H}} \mathbf{v}_{\bar{b}, \bar{k}} \mathbf{p}_{\bar{k}}^{\mathrm{H}}\right. \\
& \left.+\mathbf{Z}_{k}^{\mathrm{DL}}\right)+\mathbf{Z}_{b}^{\mathrm{UL}-2} \in \mathbb{C}^{M \times \tau}
\end{aligned}
$$

where $\mathbf{Z}_{b}^{\mathrm{UL}-2} \in \mathbb{C}^{M \times \tau}$ is the AWGN term at BS $b$ with elements distributed as $\mathcal{C N}\left(0, \sigma_{\mathrm{BS}}^{2}\right)$. Therefore, each BS $b$ obtains

$$
\begin{aligned}
& \frac{1}{\tau \sqrt{\beta^{\mathrm{UL}-2} \beta^{\mathrm{DL}}}} \mathbf{Y}_{b}^{\mathrm{UL}-2} \mathbf{p}_{k} \\
& =\sum_{\bar{b} \in \mathcal{B}} \boldsymbol{\Phi}_{b \bar{b}} \mathbf{v}_{\bar{b}, k}+\frac{1}{\tau} \sum_{\bar{k} \in \mathcal{K} \backslash\{k\}} \sum_{\bar{b} \in \mathcal{B}} \boldsymbol{\Phi}_{b \bar{b}} \mathbf{v}_{\bar{b}, \bar{k}} \mathbf{p}_{\bar{k}}^{\mathrm{H}} \mathbf{p}_{k}+\mathbf{n}_{b, k}^{\mathrm{UL}-2}
\end{aligned}
$$

where we have defined

$$
\mathbf{n}_{b, k}^{\mathrm{UL}-2} \triangleq \frac{1}{\tau \sqrt{\beta^{\mathrm{DL}}}}\left(\sum_{\bar{k} \in \mathcal{K}} \mathbf{h}_{b, \bar{k}} \mathbf{w}_{\bar{k}}^{\mathrm{H}} \mathbf{Z}_{\bar{k}}^{\mathrm{DL}}+\frac{1}{\sqrt{\beta^{\mathrm{UL}-2}}} \mathbf{Z}_{b}^{\mathrm{UL}-2}\right) \mathbf{p}_{k} .
$$

In presence of perfect channel estimation, we would have that: i) The pilot contamination in the second term of (46) is eliminated;

ii) As $\tau \rightarrow \infty$, the noise term $\mathbf{n}_{b, k}^{\mathrm{UL}-2}$ in (47) is eliminated.

Thus, (46) can be used as an estimate of $\sum_{\bar{b} \in \mathcal{B}} \boldsymbol{\Phi}_{b \bar{b}} \mathbf{v}_{\bar{b}, k}$ and each BS $b$ can obtain an estimate of $\boldsymbol{\xi}_{b, k}$ in (31) by combining the uplink signaling resources $\mathbf{Y}_{b}^{\mathrm{UL}-1}$ and $\mathbf{Y}_{b}^{\mathrm{UL}-2}$ as in (48) at the top of the page (recall that $\mathbf{N}_{b b}^{\mathrm{UL}-1} \rightarrow \sigma_{\mathrm{BS}}^{2} \mathbf{I}_{M}$ for $\tau \rightarrow \infty$ ). Finally, for each BS $b$ and for each UE $k$, the distributed joint receiver solution via OTA signaling is obtained as

$$
\begin{array}{r}
\mathbf{v}_{b, k}=\left(\mathbf{Y}_{b}^{\mathrm{UL}-1}\left(\mathbf{Y}_{b}^{\mathrm{UL}-1}\right)^{\mathrm{H}}\right)^{-1}\left(\mathbf{Y}_{b}^{\mathrm{UL}-1}\left(\mathbf{p}_{k}+\left(\mathbf{Y}_{b}^{\mathrm{UL}-1}\right)^{\mathrm{H}} \mathbf{v}_{b, k}\right)\right. \\
\left.-\tau \sigma_{\mathrm{BS}}^{2} \mathbf{v}_{b, k}-\frac{1}{\sqrt{\beta^{\mathrm{UL}-2} \beta^{\mathrm{DL}}}} \mathbf{Y}_{b}^{\mathrm{UL}-2} \mathbf{p}_{k}\right)
\end{array}
$$

which is equal to (30) for perfect CSI. The proposed dis-

\begin{tabular}{l}
\hline Algorithm 1 (Distributed-OTA) \\
\hline Data: Pilots $\left\{\mathbf{p}_{k}\right\}_{k \in \mathcal{K}}$ and $\beta^{\mathrm{UL}-2}$. \\
Initialization: Each BS $b$ initializes its combining vectors \\
$\left\{\mathbf{v}_{b, k}^{(0)}\right\}_{k \in \mathcal{K}}$; set $i=0$.
\end{tabular}

Until a predefined termination criterion is satisfied, do:

(S.0) $i \leftarrow i+1$.

(S.1) DL: Each BS $b$ transmits a superposition of pilots precoded with the corresponding combining vectors (see $\mathbf{X}_{b}^{\mathrm{DL}}$ in (15)); each UE $k$ receives $\mathbf{Y}_{k}^{\mathrm{DL}}$ in (17).

(S.2) Each UE $k$ computes its precoding vector as in (38).

(S.3) UL-1: Each UE $k$ transmits its pilot precoded with its precoding vector (see $\mathbf{X}_{k}^{\mathrm{UL}-1}$ in (5)); each BS $b$ receives $\mathbf{Y}_{b}^{\mathrm{UL}-1}$ in (7).

(S.4) UL-2: Each UE $k$ transmits $\mathbf{Y}_{k}^{\mathrm{DL}}$ precoded with $\mathbf{w}_{k} \mathbf{w}_{k}^{\mathrm{H}}$ (see $\mathbf{X}_{k}^{\mathrm{UL}-2}$ in (43)); each BS $b$ receives $\mathbf{Y}_{b}^{\mathrm{UL}-2}$ in (45).

(S.5) For each UE $k$, each BS $b$ computes its combining vectors as in (49) and updates them as in (32).

\section{End}

tributed joint receiver design via OTA signaling is formalized in Algorithm 1 (see [9] for the complexity analysis).

In this scheme, the same cross-term information that was exchanged among the BSs via backhaul links in [8] is obtained over the air at the cost of an additional uplink signaling resource. Remarkably, the amount of OTA signaling does not scale with the number of BSs $B$ or UEs $K$, and depends only on the pilot length $\tau$ and on the number of bi-directional signaling iterations. A detailed discussion on the impact of the extra signaling overhead is provided in [9], where the resulting performance loss with respect to the distributed joint receiver design via backhaul signaling is shown to be negligible for a sufficiently large resource block size (see also [11]). Lastly, the overall bi-directional signaling (consisting of DL, UL-1, and UL-2) can be easily integrated into the flexible 5G New Radio frame/slot structure; we refer to [9], [11] for more details.

\section{Numerical Results}

We consider a cell-free scenario where $B=64 \mathrm{BSs}$, each equipped with $M=8$ antennas, are placed on a square grid with distance between neighboring BSs of $30 \mathrm{~m}$ and height of $10 \mathrm{~m}$; furthermore, $K=64 \mathrm{UEs}$, each equipped with $N=2$ antennas, are randomly dropped in the same area. Each channel is generated as $\operatorname{vec}\left(\mathbf{H}_{b, k}\right) \sim \mathcal{C N}\left(0, \delta_{b, k} \mathbf{I}_{M N}\right)$, where $\delta_{b, k}[\mathrm{~dB}] \triangleq-30.5-36.7 \log _{10}\left(d_{b, k}\right)$ is the large-scale fading coefficient and $d_{b, k}$ is the distance between BS $b$ and UE $k$. The maximum transmit power of the BSs in the downlink pilot-aided channel estimation phase is $\rho_{\mathrm{BS}}=0 \mathrm{~dB}$, whereas $\rho_{\mathrm{UE}}=-10 \mathrm{~dB}$. Lastly, the AWGN power at the BSs and at the UEs is fixed to $\sigma_{\mathrm{BS}}^{2}=\sigma_{\mathrm{UE}}^{2}=-125 \mathrm{~dB}$. As performance metric, 


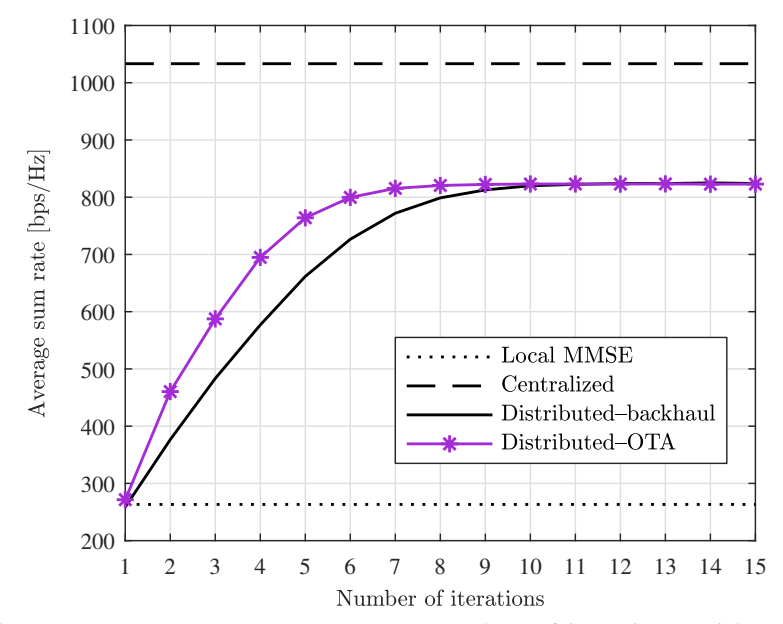

Figure 1: Average sum rate versus number of iterations with orthogonal pilots.

we evaluate the average sum rate obtained via Monte Carlo simulations with $10^{3}$ independent UE drops. In the following, we compare the distributed joint receiver design via OTA signaling described in Section V (Distributed-OTA) with the local MMSE receiver (Local MMSE), the centralized joint receiver design described in Section IV-A (Centralized), and the distributed joint receiver design via backhaul signaling described in Section IV-B (Distributed-backhaul). For the latter, we assume that the backhaul signaling introduces a delay of one iteration in the CSI exchange among the BSs (as in [9]).

Figure 1 illustrates the average sum rate versus the number of iterations considering orthogonal pilots (i.e., only the channel estimation noise is present). Remarkably, both the Distributed-OTA and the Distributed-backhaul achieve an average sum rate three times as large as the one obtained with the Local MMSE while reaching more than $80 \%$ of the performance of the Centralized. In particular, the latter gap is due to the fact that, for fixed precoding vectors, the distributed joint receiver solution requires more than one update per iteration (among the BSs) to converge to the centralized joint receiver solution. Moreover, the Distributed-OTA converges after just $7-8$ iterations and reaches about $95 \%$ of its final value after just 5 iterations. Here, the slower convergence of the Distributed-backhaul can be ascribed to the delayed backhaul update.

Assuming non-orthogonal random pilots, Figure 2 shows the average sum rate versus the pilot length; here, for the Centralized, we impose $\left\{\mathbf{P}_{k}^{\mathrm{H}} \mathbf{P}_{k}=\tau \mathbf{I}_{N}\right\}_{k \in \mathcal{K}}$ (i.e., the antennaspecific pilots within each UE $k$ are orthogonal). We observe that the Centralized is very sensitive to imperfect channel estimation as it relies on a single pilot-aided CSI acquisition. On the other hand, as detailed in [9], the distributed schemes are considerably more robust to both pilot contamination and AWGN as they involve several pilot-aided CSI acquisitions.

\section{CONCLUSIONS}

This paper proposes a distributed joint receiver design for uplink cell-free massive MIMO. An OTA signaling mechanism similar to the one proposed in [9] for the downlink scenario is

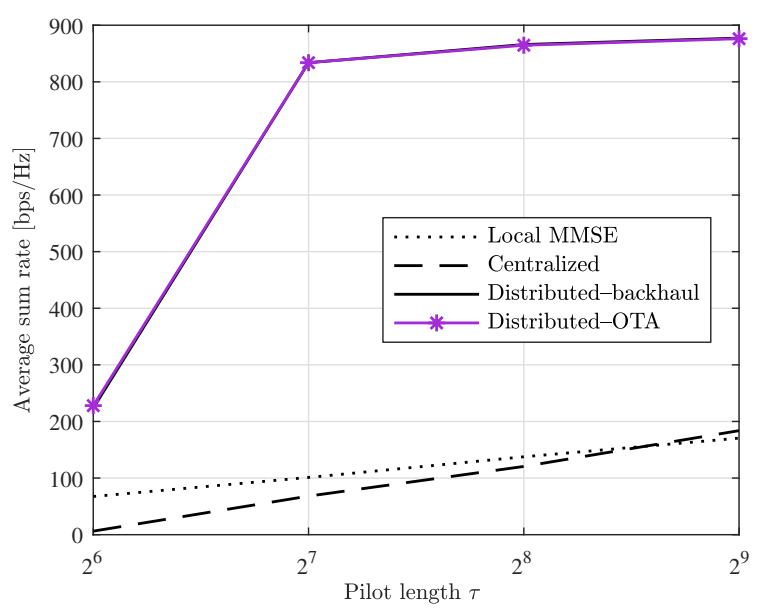

Figure 2: Average sum rate versus pilot length with non-orthogonal random pilots.

adopted to avoid CSI exchange among the BSs via backhaul signaling. Notably, the amount of OTA signaling does not scale with the number of BSs or UEs. Numerical results show very fast convergence and remarkable performance gains in terms of average sum rate with respect to the local MMSE receiver, which certainly justify the presence of extra uplink signaling.

\section{REFERENCES}

[1] H. Q. Ngo, A. Ashikhmin, H. Yang, E. G. Larsson, and T. L. Marzetta, "Cell-free massive MIMO versus small cells," IEEE Trans. Wireless Commun., vol. 16, no. 3, pp. 1834-1850, Mar. 2017.

[2] G. Interdonato, E. Björnson, H. Q. Ngo, P. Frenger, and E. G. Larsson, "Ubiquitous cell-free massive MIMO communications," EURASIP J. Wireless Commun. and Networking, vol. 2019, pp. 197-209, Aug. 2019.

[3] J. Zhang, S. Chen, Y. Lin, J. Zheng, B. Ai, and L. Hanzo, "Cell-free massive MIMO: A new next-generation paradigm," IEEE Access, vol. 7 , pp. 99878-99888, 2019.

[4] T. L. Marzetta, "Noncooperative cellular wireless with unlimited numbers of base station antennas," IEEE Trans. Wireless Commun., vol. 9, no. 11 , pp. $3590-3600$, Nov. 2010

[5] S. Buzzi, C. D'Andrea, A. Zappone, and C. D'Elia, "User-centric 5G cellular networks: Resource allocation and comparison with the cellfree massive MIMO approach," IEEE Trans. Wireless Commun., vol. 19, no. 2, pp. 1250-1264, Feb. 2019.

[6] E. Nayebi, A. Ashikhmin, T. L. Marzetta, H. Yang, and B. D. Rao, "Precoding and power optimization in cell-free massive MIMO systems," IEEE Trans. Wireless Commun., vol. 16, no. 7, pp. 4445-4459, July 2017.

[7] E. Björnson and L. Sanguinetti, "Making cell-free massive MIMO competitive with MMSE processing and centralized implementation," IEEE Trans. Wireless Commun., vol. 19, no. 1, pp. 77-90, Jan. 2020.

[8] J. Kaleva, A. Tölli, M. Juntti, R. A. Berry, and M. L. Honig, "Decentralized joint precoding with pilot-aided beamformer estimation," IEEE Trans. Signal Process., vol. 66, no. 9, pp. 2330-2341, May 2018.

[9] I. Atzeni, B. Gouda, and A. Tölli, "Over-the-air distributed precoding design for cell-free massive MIMO systems," (Submitted), 2020.

[10] Q. Shi, M. Razaviyayn, Z.-Q. Luo, and C. He, "An iteratively weighted MMSE approach to distributed sum-utility maximization for a MIMO interfering broadcast channel," IEEE Trans. Signal Process., vol. 59, no. 9, pp. 4331-4340, Sept. 2011.

[11] A. Tölli, H. Ghauch, J. Kaleva, P. Komulainen, M. Bengtsson, M. Skoglund, M. Honig, E. Lahetkangas, E. Tiirola, and K. Pajukoski, "Distributed coordinated transmission with forward-backward training for 5G radio access," IEEE Commun. Mag., vol. 57, no. 1, pp. 58-64, Jan. 2019.

[12] G. Scutari, F. Facchinei, P. Song, D. P. Palomar, and J.-S. Pang, "Decomposition by partial linearization: Parallel optimization of multiagent systems," IEEE Trans. Signal Process., vol. 62, no. 3, pp. 641-656, Feb. 2014. 